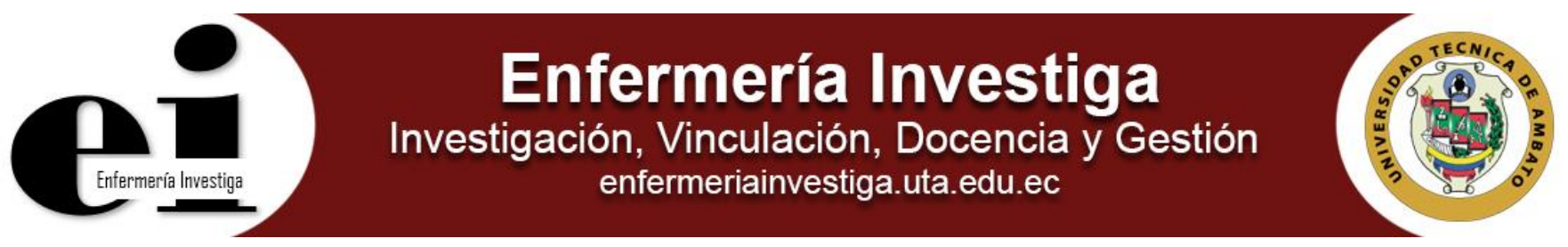

DOI: http://dx.doi.org/10.29033/ei.v2n4.2017.07

Artículo de revisión

\title{
Microbiota intestinal, probióticos y prebióticos
}

Intestinal microbiota, probiotics and prebiotics

\author{
Carlos David Castañeda Guillot ${ }^{1,2}$
}

\author{
${ }^{1}$ Universidad Regional Autónoma de los Andes - Facultad de Ciencias Médicas - Carrera de Medicina - Ambato - Ecuador \\ 2 Universidad de Ciencias Médicas de la Habana - Faculta de Medicina Calixto García - Carrera de Medicina - La Habana - Cuba
}

Castañeda GCD. Microbiota intestinal, probióticos y prebióticos. Enferm Inv (Ambato). 2017; 2(4):156-160.

2477-9172 / 2550-6692 Derechos Reservados ( 2017 Universidad Técnica de Ambato, Carrera de Enfermería. Este es un artículo de acceso abierto distribuido bajo los términos de la Licencia Creative Commons, que permite uso ilimitado, distribución y reproducción en cualquier medio, siempre que la obra original es debidamente citada.

\section{Historia:}

Recibido: 11 noviembre 2017

Revisado: 22 noviembre 2017

Aceptado: 29 noviembre 2017

Palabras Claves: Microbiota intestinal; probióticos; prebióticos

\section{Keywords: Intestinal} microbiota; probiotics: prebiotics

\begin{abstract}
Resumen
La microbiota intestinal es el elemento fundamental del "Ecosistema Intestinal", cuyas acciones tienen un efecto beneficioso para la salud, al participar de forma activa en los procesos de digestión de nutrientes asociado a sus mecanismos sobre la homeostasis y la inmunidad del intestino. Los probióticos y prebióticos juegan un rol decisivo en la modulación de la microbiota intestinal y han demostrado sus beneficios para el tratamiento de distintas enfermedades intestinales y extraintestinales. Se realizó una búsqueda en bases documentales de PubMed, SciELO y Latindex de publicaciones relacionadas con la microbiota intestinal, probióticos y prebióticos hasta el 30 junio 2017. Son actualizados los criterios relacionados con las funciones de la microbiota intestinal, el proceso de implantación a partir del nacimiento, el desarrollo acelerado en su composición, los distintos factores que influencian en su diversidad y estabilidad para mantener un estado de eubiosis en el curso de la vida. El desequlibrio de la misma (disbiosis) es un elemento de importancia en la producción de afecciones intestinales y extraintestinales agudas y crónicas. Se revisaron los probióticos y prebióticos, sus ventajas, tipos, indicaciones y las formas de obtención de los prebióticos. El papel de la microbiota intestinal como órgano metabólico por sus múltiples funciones, en especial la homeostasis e inmunidad intestinal y sus beneficios para la salud son revisados. La participación de los probióticos y prebióticos como terapéutica y sus efectos en enfermedades intestinales y extra-intestinales relacionadas con la microbiota y su modulación son examinados por su importancia en la práctica médica.
\end{abstract}

\begin{abstract}
The intestinal microbiota is the fundamental element of the "Intestinal Ecosystem", whose actions have a beneficial effect on health, by actively participating in the processes of nutrient digestion associated with its mechanisms on homeostasis and immunity of the intestine. Probiotics and prebiotics play a decisive role in the modulation of the intestinal microbiota and have shown their benefits for the treatment of various intestinal and extra-intestinal diseases. We searched the PubMed, SciELO and Latindex documentary databases for publications related to intestinal microbiota, probiotics and prebiotics until June 30,2017 , and related books. The criteria related to the functions of the intestinal microbiota, the implantation process from birth, the accelerated development of its composition, the different factors that influence its diversity and stability to maintain a state of eubiosis in the course of lifetime. The deficit of the same (dysbiosis) is an important element in the production of acute and chronic intestinal and extra-intestinal affections. We review the probiotics and prebiotics, their advantages, types, indications and the ways of obtaining the prebiotics. The role of the intestinal microbiota as a metabolic organ due to its multiple functions, especially intestinal homeostasis and immunity and its health benefits are reviewed. The participation of probiotics and prebiotics as a therapeutic and their effects on intestinal and extra-intestinal diseases related to the microbiota and its modulation are examined for their importance in medical practice.
\end{abstract}




\section{Introducción}

El término "flora intestinal" usado antaño, pasó a desuso desde finales de la década de los años 90 s, al ser sustituido por "microbiota intestinal", al consider que la población bacteriana que la formaba, constituida por población bacteriana comensal, indígena o saprofita, no era solamente un conglomerado de bacterias, sino un conjunto de microorganismos vivos con acciones efectivas de importancia sobre la salud humana ${ }^{1,2}$.

En la actualidad se reconoce a la "microbiota intestinal" como la población de microorganismos que habitan en el intestino y ayudan a digerir y asimilar los nutrientes que son ingeridos, incluido vitaminas, fibras y azúcares, lo que evidencia la activa participación de sus constituyentes. El mayor número se localiza en la porción distal del tubo digestivo, en el colon ${ }^{3}$. Este concepto varió en relación antaño, en los años previos al nuevo milenio.

Entre las principales acciones reconocidas de la microbiota intestinal sobresale su capacidad de intervenir en los eventos de homeostasis e inmunidad del intestino ${ }^{4-6}$.

Se realiza búsqueda en las bases documentales de PubMed, SciELO y Latindex de publicaciones relacionadas con la microbiota intestinal, probióticos y prebióticos hasta 30 junio 2017, y libros afines.

El objetivo de este artículo es realizar una puesta al día de los beneficios para la salud de la microbiota intestinal y los efectos de los probióticos y prebióticos en los eventos de disbiosis (desequilibrio de la microbiota intestinal) y modulación intestinal de la constitución de su microbiota en el curso de la vida.

\section{Desarrollo}

\section{Microbiota intestinal}

A finales del pasado siglo $X X$ se postulaba la microbiota intestinal estaba constituida entre 500 y 1000 microorganismos, sin embargo, los estudios más recientes realizados con la técnica de biología molecular han determinado en años recientes está formada entre 15000 a más de 35000 especies, según el método seguido ${ }^{7}$.

Hoy día se reconoce la existencia de cuatro divisiones o phylum como constituyentes de la microbiota intestinal: Firmicutes (gram positivos), Bacteroidetes (gram negativo), Actinobacterias (gram positivo) y Protobacterias (gram negativo $)^{8}$. Los hongos y Archaea pueden ser también residentes, su población es de $1 \%$ del total ${ }^{9}$. La microbiota presenta un predominio de Firmicutes y Bacteriodetes, que representan entre el 90 al $99 \%$, en humanos y ratones, la cual varía en distintas enfermedades ${ }^{10}$.

La colonización de la microbiota intestinal se inicia al momento del nacimiento y días posteriores, y está influenciada por factores de la forma de nacimiento (parto normal versus cesárea), tipo de alimentación (lactancia materna versus lactancia artificial), tiempo de gestación y peso al nacimiento (normal versus bajo peso y prematuridad), y condiciones del medio ambiente y estilo de vida (países con buen estado económico versus países en vías de desarrollo). Alrededor de los dos a tres años de edad, y en ocasiones más tarde, hasta los cinco años de edad, es que se alcanza el pleno desarrollo en la constitución de la microbiota intestinal, la cual permanecerá estable en la infancia y adultez, al alcanzar una adecuada diversidad. Estos factores son decisivos para el establecimiento de eubiosis o disbiosis a largo plazo y su repercusión en la aparición de enfermedades intestinales y extra-intestinales. Según las variaciones de los factores mencionados el proceso de colonización varía con la consiguiente estabilidad en la salud o con la aparición de distintas enfermedades, como causas autoinmunes (colitis ulcerosa y enfermedad de Crohn), alérgicas (alergia proteínas de la leche de vaca y eczema) y metabólicas (obesidad y diabetes mellitus tipo 2), entre las más frecuentes ${ }^{1,3,5-8}$.

La microbiota intestinal es estable en el tiempo en el adulto sano. En cada sujeto, aun cuando exista estabilidad en el transcurso de la vida, varía la diversidad, la cual aumenta en el curso de los años. Los antibióticos, enfermedad aguda temporal y viajes al extranjero son factores que pueden producir alteraciones en la composición de la microbiota ${ }^{1}$.

El concepto de "Ecosistema Intestinal" desarrollado desde la década de los años 60s del pasado siglo y enarbolado por el autor por su interrelación dialéctica entre la regulación cualitativa de la microbiota intestinal y su microbioma, expresado en las interacciones a que están sometidos los microorganismos que la constituyen, los sustratos digestivos y el sistema inmune; representa un elemento de trascendencia para la comprensión de la fisiología del intestino $y$ ha permitido la microbiota intestinal sea interpretada como un "órgano metabólico"1.

Las funciones de la microbiota intestinal son metabólicas, tróficas y protección. En la tabla 1 se resumen las mismas ${ }^{1}$.

Tabla 1. Funciones de la microbiota intestinal.

\begin{tabular}{lll}
\hline Metabolismo & y & $\begin{array}{l}\text { Fermentación de residuos no } \\
\text { digestivos de la dieta y moco } \\
\text { intestinal. } \\
\text { Energía, con producción ácidos } \\
\text { grasos cadena corta. }\end{array}$ \\
& $\begin{array}{l}\text { Producción de vitaminas (K y ácido } \\
\text { fólico) } \\
\text { Absorción de iones. }\end{array}$ \\
\hline Trófica & $\begin{array}{l}\text { Control de la proliferación celular } \\
\text { epitelial y su diferenciación. }\end{array}$ \\
\hline Protección & Efecto de barrera: protección contra \\
& los antígenos y gérmenes \\
& patógenos.
\end{tabular}

Fuente: Tomado de: Castañeda C. capítulo 2. Microboiota intestinal. En: Ecosistema Intestinal. 2da. Ed. Editorial Mendieta, Quito, $2014^{1}$.

\section{Probióticos}

Los probióticos surgen en la década de los años 60 s con la conceptualización inicial realizada por Lily y Stilwell ${ }^{11}$, pero fue Parker $^{12}$ en 1974, quién lo define como hoy son conocidos, aunque hacía mucho tiempo antes eran consumidos con la ingestión de alimentos ancestrales, como el yogur o leches fermentadas, siguiendo los argumentos enarbolados desde inicio del siglo XX en París, por el premio Nobel, de Medicina en 1908, el judío ucraniano Elier Metchnikoff, donde ocupaba la sub-dirección del Instituto Pasteur; acerca el efecto beneficioso de las bacterias vivas productoras de ácido láctico de los géneros Lactobacillus y Bifidum con que se preparaba las leches fermentadas y el yogur para el consumo humano, representativo del efecto probiótico de los mismos. Metchnikoff había publicado en 1907 una tesis donde reportaba la influencia de las bacterias lácticas en la microbiota intestinal, pues en su opinión las mismas influenciaban sobre la longevidad de los pobladores de la región de los Balcanes, en Europa del Este, lo que evidenciaba sus beneficios para la salud ${ }^{13}$. 
El yogur es un alimento altamente saludable para el humano, ya sea lactante, niño mayor, adulto y anciano; por su contenido en proteínas, vitaminas y minerales, en especial de vitamina $D$ y calcio respectivamente en ambos últimos; y cultivos vivos, lo que le aporta su efecto probiótico $^{14}$ (tabla 2).

Tabla 2. Definición de probióticos y prebióticos.

\begin{tabular}{|c|c|}
\hline Término & Definición \\
\hline Probióticos & $\begin{array}{l}\text { microorganismos vivos que después de } \\
\text { su ingestión en un número específico, } \\
\text { ejercen beneficios para la salud del } \\
\text { huésped, más allá de aquellos que son } \\
\text { inherentes a la nutrición básica }{ }^{15} \text {. }\end{array}$ \\
\hline Prebióticos & $\begin{array}{l}\text { sustancias de la dieta (fundamentalmente } \\
\text { polisacáridos no amiláceos y } \\
\text { oligosacáridos no digeribles por enzimas } \\
\text { humanas) que nutren a grupos } \\
\text { seleccionados de microorganismos que } \\
\text { habitan en el intestino, favoreciendo el } \\
\text { crecimiento de bacterias beneficiosas } \\
\text { sobre las nocivas }{ }^{16} \text {. }\end{array}$ \\
\hline
\end{tabular}

2014 y

\section{Rol de probióticos y prebióticos}

\section{Probióticos}

Su aplicación ha sido respaldada por variados mecanismos en distintas edades. A continuación, se expresan las principales evidencias de su administración, siguiendo los criterios de la medicina basada en la evidencia ${ }^{17}$.

\section{Probióticos en la infancia}

Las experiencias acumuladas por la administración de probióticos están respaldada por estudios a doble ciego, aleatorizados y meta-análisis que han permitido evidenciar su eficacia en afecciones intestinales y extraintestinales ${ }^{18,19}$. Su indicación como adjuvante con las sales de rehidratación oral en la diarrea aguda infantil es recomendada por los resultados de investigaciones latinoamericanas ${ }^{20}$ y europeas ${ }^{21}$, en especial la diarrea de causa viral producida por rotavirus, al reducir en un día el proceso diarreico; es eficiente en la diarrea asociada a antibióticos y asociada como adjuvante del tratamiento ${ }^{22}$; en el tratamiento y prevención de la recidiva de la infección por Clostridium difficile ${ }^{23}$; como preventivo y terapéutica en los recién nacidos de bajo peso o prematuridad con enterocolitis necrosante ${ }^{24}$ y en la prevención de alergia del recién nacido y la alergia a la leche de vaca productora de eczema ${ }^{25}$. Estas indicaciones están avaladas por nivel de evidencia A.

\section{Probióticos en el adulto}

En presencia de trastornos de la colonización y como coadyuvante en determinadas específicas condiciones son usados con provecho ${ }^{26,27}$. Además de la diarrea aguda, se ha demostrado su utilidad en la bolsitis de pacientes operados de colitis ulcerosa, al producir remisión del proceso inflamatorio y prevenir la recurrencia, con una disminución significativa de recaídas ${ }^{28}$; como coadyuvante del tratamiento en la diarrea asociada a antibióticos y recurrencia de la infección por Clostridium dfficile ${ }^{29} ; y$ en presencia de Helicobacter pyloris se ha precisado reducción de su adherencia a la mucosa del estómago ${ }^{30,31}$.

\section{Probióticos en el anciano}

La contribución de su administración para estimular la actividad inmunitaria es evidente, la cual se reduce al detener la administración del probiótico. El consumo de yogur conteniendo probiótico dos a tres veces al día normaliza el tránsito intestinal en los sujetos en quiénes presentan el antecedente de ser prolongado, por padecer de estreñimiento. En la intolerancia a la lactosa del anciano es recomendada la administración de probiótico. El potencial uso de yogur en la senilidad no ha sido estudiado a plenitud. Nuevas investigaciones permitirán determinar acerca de sus indicaciones con beneficiosos efectos. ${ }^{32}$

Indicaciones de los probióticos ${ }^{13}$

- Mejora el estado de equilibrio de la microbiota y salud intestinal.

- $\quad$ Reduce la severidad de la diarrea aguda en la infancia.

- Previene la diarrea asociada a antibióticos.

- Reduce los síntomas de intolerancia a la lactosa.

- Reduce los síntomas del intestino irritable.

- Estimula el sistema inmune.

- Sintetiza y mejora la biodisponibilidad de los nutrientes.

- Disminuye la presencia de eccema.

- Reduce el riesgo de cáncer de colon.

Los probióticos son clasificados en bacterianos y de levadura. A los bacterianos corresponden los géneros de Lactobacillus, Bifidobacterium, Streptococcus, Lactococcus, Enterococcus y Bacillus y cepas no patógenas de E. coli (Nissle 1917) que compiten con su homólogo patógeno; y por levadura sólo existe el Saccharomyces boulardii. Existen múltiples probióticos, pero no todos son iguales y su indicación varía según la afección y en dependencia del tipo de probiótico, por género, especie y cepa (con identificación numeral $)^{13}$ (tabla 3).

Hay una serie de otras indicaciones en los que los probióticos han sido usados, pero los niveles de evidencia son B y C. Entre ellos se encuentran la infección por Clostridium difficile, síndrome del intestino irritable, colitis ulcerosa, enfermedad de Crohn y en la colitis por radiación ${ }^{13}$.

\section{Prebióticos}

Los prebióticos se conceptúan en 1995 por Gibson y Roberfroid $^{33}$, pero esta definición es revisada por el propio Roberfroid en 2007, estableciendo "son ingredientes selectivamente fermentados por la microbiota intestinal y que provocan en ella cambios en su composición y actividad, con efectos beneficiosos para la salud del individuo" 34 . En Japón, Yazawa ${ }^{35}$, ya en 1978 postulaba sus efectos sobre las bifidobacterias, favoreciendo su crecimiento.

En 2016 un panel de expertos auspiciados por la Asociación Científica Internacional de Probióticos y Prebióticos revisó la definición y alcance de los prebióticos, estableciendo son "sustrato que es utilizado selectivamente por los microorganismos del huésped, confiriendo beneficios para la salud", que incluye también sustancias que no son carbohidratos $^{36}$. 
Tabla 3. Probióticos: Indicaciones y tipos.

\begin{tabular}{ll}
\hline Indicaciones: Evidencia A & Tipo de probiótico (género, especie y cepa) \\
\hline Diarrea aguda infecciosa en niños & Lactobacillus rhamnosus GG \\
& Saccharomyces boulardii CNCM I-745 \\
& Lactobacillus reuteri DSM 17938 \\
& Lactobacillus acidophilus+Lactobacillus casei \\
& Lactobacillus bulgaricus+Bifdobacterium bifidum+ \\
\hline Diarrea asociada antibióticos & Streptococcus thermophilus \\
\hline Modulación inmunológica & Saccharomyces boulardii CNCM I-745 \\
& Lactobacillus rhamnosus HN001 \\
& Lactobacillus acidophilus CL1285 \\
& Lactobacillus plantarum 299v (DSM 9843) \\
& Bifidobacterium lactis \\
& Lactobacillus casei cepa Shirota \\
\hline Afecciones alérgicas & Lactobacillus rhamnosus GG \\
con eccema & Lactobacillus johnsonii \\
\hline Bolsitis en pacientes con colitis ulcerosa & Lactobacillus rhamnosus GG \\
\hline
\end{tabular}

The Health Benefits ofrol

Fuente: Modificado de "Proceedings of the Symposium. The Heath

La participación de los prebióticos en el equilibrio y diversidad de la composición de la microbiota intestinal es un decisivo exponente de los efectos de estos ingredientes alimenticios en la producción de cambios específicos favorables en la nutrición de la microbiota y el trofismo de la mucosa del colon con sus consiguientes efectos sobre la salud $^{37}$.

La denominación de prebiótico debe cumplir los criterios de resistencia a la digestión en el tracto digestivo superior, fermentación de la microbiota intestinal y beneficios para la salud del huésped. En la tabla 4 se muestran las características de los prebióticos.

Tabla 4. Rasgos de los prebióticos.

\begin{tabular}{l} 
Rasgos de los prebióticos \\
\hline Producto natural no hidrolizado ni absorbible en el tracto \\
digestivo superior. \\
Capacidad de modificar la composición de la microbiota \\
del colon tras ser selectivamente fermentada por una o \\
varias bacterias.
\end{tabular}

Capacidad de modificar la composición de la microbiota del colon tras ser selectivamente fermentada por una o varias bacterias.

La estimulación selectiva de bacterias intestinales induce beneficios para la salud.

Fuente: Tomado de: Castañeda C, Del Monte A. Prebióticos: obtención y repercusión para la salud. Ed. Mendieta, Quito. $2014^{37}$.

\section{Indicaciones de los prebióticos}

Los prebióticos han demostrado efectos favorables en el estreñimiento funcional, trastornos digestivos funcionales (síndrome intestino irritable), enfermedades inflamatorias intestinales, prevención de la diarrea asociada a Clostridium difficle (o prevención de recurrencia), tratamiento de la encefalopatía hepática, prevención del cáncer de colon, hipercolesterolemia, y absorción de calcio en el intestino delgado o el colon ${ }^{18,37}$.

\section{Distintos tipos de prebióticos}

Habitualmente en la dieta humana y animales se encuentran oligosacáridos no digeribles y polisacáridos. Los principales oligosacáridos no digeribles son: Fructo-oligosacáridos (FOS, oligofructosa e inulina), Galacto-oligosacáridos (GOS), Transgalacto-oligosacáridos (TOS) y lactulosa. Otros prebióticos, aunque menos estudiados son: Glucooligosacáridos, Malto-oligosacáridos, Xilo-oligosacáridos y Manono-oligosacáridos ${ }^{37}$. En la tabla 5 se relaciona la acción de distintos prebióticos en determinadas afecciones clínicas en humanos. También son usados en animales y peces.

Tabla 5. Prebióticos: Indicaciones, tipos y dosis.

\begin{tabular}{|c|c|c|}
\hline Afección/Enfermedad & Prebiótico & Dosis \\
\hline $\begin{array}{l}\text { Prevención de la diarrea } \\
\text { asociada } \\
\text { a Clostridium difficile (o } \\
\text { prevención } \\
\text { de recurrencia) }\end{array}$ & Oligofructosa & $\begin{array}{c}4 \text { g/tres } \\
\text { veces/ } \\
\text { día }\end{array}$ \\
\hline Síndrome intestino irritable & $\begin{array}{c}\text { Fructo- } \\
\text { oligosacárido } \\
\text { de cadena } \\
\text { corta } \\
\text { Galacto- } \\
\text { oligoscárido }\end{array}$ & $\begin{array}{c}5 \mathrm{~g} / \mathrm{día} \\
3,5 \mathrm{~g} / \mathrm{dí} a\end{array}$ \\
\hline Estreñimiento funcional & $\begin{array}{l}\text { Lactulosa } \\
\text { Oligofructosa }\end{array}$ & $\begin{array}{c}20-40 \\
\text { g/día } \\
20 \text { g/día }\end{array}$ \\
\hline Encefalopatía hepática & Lactulosa & $\begin{array}{l}45-90 \\
\text { g/día }\end{array}$ \\
\hline
\end{tabular}

A, Garisch $\mathrm{J}$ et al. World Gastroenterology Organization. Probiotics and Prebiotics. $2017^{18}$.

En la naturaleza existen múltiples fuentes de materia prima para la obtención de oligoscáridos no digeribles. Entre estas fuentes naturales está la leche (materna y de vaca), el calostro, la miel de abeja; entre los vegetales la cebolla, ajo, alcachofa, puerro, remolacha, tomate y espárragos; entre las frutas, el plátano; entre los cereales, la cebada, trigo y centeno; entre las leguminosas, los frijoles, lentejas y soya; caña de azúcar y derivados como jugo de caña de azúcar, melazas y bagazo, y el maíz ${ }^{38}$.

En el contexto del desarrollo biotecnológico, a la luz de los conocimientos desarrollados en los años recientes, los prebióticos pueden ser obtenidos por proceso industrial y laboratorio utilizando disacáridos como sustrato. Se han desarrollado tres vías ${ }^{39}$ :

1. Procedimientos de extracción directa de material vegetal.

2. Procedimientos de extracción de oligosacáridos y posterior hidrólisis química (por diferentes métodos).

3. Procedimientos combinaos mediante extracción química y biocatálisis para procesos de bioconversión enzimática. 


\section{Conclusiones}

El papel de la microbiota intestinal como órgano metabólico, sus funciones y efectos sobre la homeostasis e inmunidad resultan fundamentales para el proceso de digestión de los nutrientes y su demostrado efecto sobre la salud en el curso de la vida. El proceso de implantación, los factores participantes y los rasgos de su composición y diversidad son revisados. La repercusión de la microbiota intestinal en la disbiosis y principales enfermedades intestinales y extraintestinales con trastornos de la microbiota son expuestas. El papel de probióticos y prebióticos son conceptualizados, sus ventajas para la modulación y equilibrio de la microbiota intestinal son destacados. Se revisaron sus acciones, indicaciones de probióticos y prebióticos y las formas de obtención de los prebióticos.

\section{Conflicto de intereses}

Ninguno declarado por los autores.

\section{Agradecimientos}

Ninguno declarado por los autores.

\section{Referencias}

1. Castañeda C. Capítulo 2. Microbiota intestinal. En: Ecosistema Intestinal. 2da. Ed. Ed. Mendieta, Quito 2014. P.21-34.

2. Jandhyala SM, Taludar R, Subramanyam C, Vuyuru $H$, Sasikala M, Nageshwar-Reddy D, Role of the normal gut microbiota. World $J$ Gastroenterol 2015 Aug 7:21(29):8787-803. doi10.378/wjg. v21.i29.8787. Published online 2015 Aug 7.

3. Hao WL, Lee YK. Microflora of the tract gastrointetinal a review. Methods Mol Biol 2004; 268:491-502.

4. Wu HJ, Wu E. he role of gut microbiota in immune homeostasis and autoimmunity. Gut Microbes 2012; 3(12):4-14

5. Madsen K, Park H. Immunologic response in the host. Chapter 26 en: The Microbiota Gastrointestinal Pathophysiology. Implications for human, prebiotics, probiotics, and dysbiosis. Ed. Floch M, Randel y, Walker WA.1er ed. Academic Press. Boston 2016. P. 223-41.

6. Castañeda C. Microbota intestinal e inmunidad intestinal. 2016; 18(3). Supl 3 (septiembre-diciembre).

7. Frank DN, St Armand AL, Feldman RA, Boedeker EC, harpaz N, pace NR. Molecular-phylogenetic characteriztion of microbial commuity imbalances in human inflammatory bowel diseases. Proc Natl Acad Sci USA 2007; 104:13780-5.

8. Stecher B, Hardt WD. The role of microbiota in infectious disease. Trend Microbiol 2008-Mar 18(3):107-14. doi: 10.1016/.tim.2007.12.008.

9. Rajilić-Stojanović M, de VosWM. Thefirst 1000 cultured species of the human intestine nd its regulation. J Clin Gastroenterol 2007;41:S2-S6.

10. Icaza-Chávez ME, Microbiota intestinal en la salud y la enfermedad. Rev Gastroenterol Mex. 2013 oct-dic; 78(4):240-8.

11. Lily DM, Stilwell RH. Probiotics: growth promoting factors produced by microorganisms. Science 1965; 147:747-8.

12. Parker RB. Probiotics, the other half of antibotic stry. Amer Nutr Health 1974; 29:4-8.

13. Castañeda C. Capítulo 6. Probióticos. En Ecosistema Intestinal. 2da. ed. Quito. Editorial Mendieta. 2014. P 95-104.

14. Mu Chen W, Sun Q, Giovanucci E, Mozaffarian D, Manson JE, Willet WC et al. Dairy consumption and risk of type 2 diabetes: 3 cohorts of US adults and updated meta-analysis. Medicine 2014; $12: 215$

15. Hill C, Guarner F, Reid G, Gibson GR, Merenstein DJ, Pot B et al. Epert consensus document. The International Scientific Association for Probiotics and Prebiotics consensus statement on the scope and aprooppriate use of the term probiotic. Nat Rev Gastroenterol Hepatol 2014; 11:506-14.

16. Bindels LB, Delzeen NM, Cani PD, Walter J. Towards a more comprehensive concept for prebiotics. Nat Rev Gastroenterol Hepatol 2015 May;12(5):303-10. doi: 10.1038/ngastro.2015.47

17. Castañeda C, Martínez R. La medicina basada en la evidencia. GASTROHNUP 2014; 16(2):101-11.

18. Guarner F, Sanders ME, Eliakim R, Fedorak R, Gangl A, Garisch J et al. World Gastroenterology Organization. Probiotics and Prebiotics 2017. http:77www.worldgastroenterology.org/guidelines/global-

guidelines/probiotic-and-prebiotics/probiotic-and-prebiotics-spanish

19. Alvarez-Calatayud G, Pérez-Moreno J, Tolin M, Sánchez C. Aplicaciones clínicas del empleo de probióticos en pediatría. Nutrición hospitalaria 2013 may/jun; 28(3):569-74

20. Cruchet S, Funes R, Maruy A, Hebel E, Palacios J, Medina F et al. Uso de probióticos en gastroenterología pediátrica: revisión de la literatura y recomendaciones de expertos latinoamericanos. Pediatric Drugs 2015; 12:199-216.

21. Szajewska H, Guarino A, Hojsak I. Use of probiotics for management of acute gastroenteritis: a position paper by the ESPGHAN Working Group for probiotics and prebiotics. J Pediatr Gast Nutr 2014 Apr;58 (4):531-9.

22. Szajeweka H, Canani RB, Guarino A, Hojsak I, Indrio F, Kolacek F e al, on Behalf of the ESPGHAN Working Group for Probiotics/Prebiotics. Probiotics for the prevention of AntibioticAssociated Diarrhea in children. J Pediatr Gast Nutr 2016 March; 62 (3):495-506.

23. Tamma PD, Sandora PJ. Clostridium difficile infection in children Current state and unanswered questions. J Ped Infect Dis Soc. 2012; 1(3):230

24. Alvarez-Calatayud G, González-Pacheco N, Seguridad en el empleo de probióticos en la prevención de enterocolitis necrosante en la prematuridad.

1. El Probiótico. https//www.el probiótico.com/seguridad-pobóticosprevención-enterocolitis-prematuros/

25. Boyle RJ, Mah LJ, Chen A, Kikivuori S, Licciardi PV, Robins Browne $\mathrm{RM}$, Mah LJ et al. Lactobacillus GG treatment during pregnancy for the prevention of eczema. A randomized controlled trial. Allergy 2011; 66(4):509-16.

26. Valdovino MA, Montijob E, Abreuc AT, Hellerd S, González-Garaye A, Bacarrezaf $D$ et al. Consenso mexicano sobre probiótcos y prebióticos en gastroenterología. Rev Mex Gastronterología 2017, Apr-Jun; 82(2):156-78.

27. Sanders ME, Guarner F, Guerrant R, Holt PR, Quigley E, Sandor RB et al. An update on the use and investigation of pobiotics in health and disease. Gut 2013 May;62(5):787-96. Doi:10.1136/gut/nl-2012302504

28. Gionchetti P, Rizzeto F, Morselli C, Poggioli G, Tambasco R, Calabrese $\mathrm{C}$ et al. High-dose probiotics for the treatment of active pouchitis. Dis Colon Rectum 2007 Dec;50(12):2075-82.

29. Johnson S, Maziade P-J, McFarland LV, Trick W, Donskey C, Currie $B$ et al. Is primary prevention of Clostridium difficile infection posible with specific proiotics? Int $J$ Infect Dis IJID Off Publ Int Soc Infect Dis $2012 \mathrm{Nov}$;1611) cc786-792

30. Zheng Z, Liu L, Mei Z. Lactobacillus containing probiotic supplementation increases Helicobacter pylori eradication rate: evidence from a meta-anlaysis. Rev Esp Enf Dig 2013 Sep 105 (8):445-531)

31. Du YQ, Su T, Fan JG, Lu YX, Zheng P, Li GHET AL. Adjuvant probiotics improve eradication efect of triple therapy for Helicobacter pylori infection. World J Gastroenterol 2012 Nov; 18(43):6302-7. DOI: 10.3748/wjg.v18.143.6302

32. Floch M. Reommendations for probiotic use by patients. Proceedings of the Symposim The Health Benefits of Probiotics. American Academy of Physician Assistants $37^{\text {th }}$ Annual PA Conference, EUA $2017 . \quad \mathrm{http}: / / \mathrm{www}$.aapa.org/education-and-ertificationcontinuingmedical-education/webcasts

33. Gibson GR, Roberfroid MB. Dietary modulation of the human colonic microbiota - introducing the concept probiotics. J. Nutrition 1995; 125:1401-12.

34. Roberfroid MB. Prebioics: The concept revisited. J Nutrition 2007; 137:830-7.

35. Yazawa K, Imai K, Tamura Z. Oligosaccharides and polysaccharides specifically utilizable by bifidobacteria. Chem Pharmacol Bull 1978; 26:3306-11

36. Gibson GR, Hutkins R, Sanders ME, Prescott SL, Reymer RA, Salminen SJ et al. Expert consensus document: The International Scientific Association for Probiotics and Prebiotics (ISAPP) consensus statement on the definition and scope of prebiotics. Nat Rev Gastroenterol Hepatol 2017 Aug; 14(8):491-502.

37. Castañeda C, Del Monte A. Capítulo 3. Prebióticos y su repercusión en la salud. En: Prebióticos. Su obtención y repercusión para la salud. Ed. Mendieta, Quito 2014. P 35-44.

38. Castañeda C, Del Monte A. Capítulo 4. Oligosacáridos no digeribles. En: Prebióticos. Su obtención y repercusión para la salud. Ed. Mendieta, Quito 2014. P45-49.

39. Castañeda C, Del Monte A. Capítulo 5. Obtención de prebióticos. En Prebióticos. Su obtención y repercusión para la salud. Ed. Mendieta, Quito 2014. P 51-54. 